\title{
High-energy neutrino as observational signature of massive black hole formation
}

\author{
V. Berezinsky ${ }^{1,2}$ and V. Dokuchaev ${ }^{2}$ \\ ${ }^{1}$ Laboratori Nazionali del Gran Sasso, INFN, 67010 Assergi (AQ), Italy \\ e-mail: berezinsky@lngs .infn. it \\ 2 Institute for Nuclear Research of the Russian Academy of Sciences, 60th October Anniversary Prospect 7a, 117312 Moscow, Russia \\ e-mail: dokuchaev@inr.npd.ac.ru
}

Received 8 November 2004 / Accepted 25 March 2006

ABSTRACT

\begin{abstract}
We describe the formation of a seed massive black hole (MBH) inside a supermassive star (SMS) in a distant galactic nucleus. The short-lived SMS is naturally formed due to collision destructions of normal stars in the evolving galactic nucleus. The neutron stars (NSs) and stellar-mass black holes form a compact self-gravitating subsystem deep inside a SMS. This subsystem is shortlived in comparison with a host SMS and collapses finally into the MBH. Just before gravitational collapse of compact subsystem the frequent NS collisions are accompanied by the generation of numerous ultra-relativistic fireballs. A combined ram pressure of multiple coexisting fireballs produces a quasi-stationary rarefied cavity in the central part of SMS. The protons are accelerated in the fireballs and by relativistic shocks in the cavity. All secondary particles, produced in collisions, except the high-energy neutrinos are absorbed in the SMS interiors. An estimated high-energy neutrino signal from this hidden source can be detected by the neutrino telescope with an effective area $S \sim 1 \mathrm{~km}^{2}$ providing the evidence for MBH formation in a distant galactic nucleus. A corresponding lifetime of this high-energy hidden neutrino source is $\sim 0.1-1 \mathrm{yr}$.
\end{abstract}

Key words. neutrinos - black hole physics - acceleration of particles

\section{Introduction}

The origin of massive black holes (MBHs) in the galactic nuclei is a long standing problem. In this paper we describe a "natural" scenario of a seed MBH formation in the process of dynamical evolution of a central stellar cluster in the galactic nuclei. We demonstrate that high-energy neutrinos may be the observational signature of $\mathrm{MBH}$ formation.

High-energy (HE) neutrino radiation from astrophysical sources is accompanied by other types of radiation, most notably by the HE gamma-radiation. This HE gamma-radiation put an upper limit on the neutrino flux emitted by the neutrinotransparent astrophysical sources. For example, if neutrinos are generated by the interaction of HE protons with low energy photons in the extragalactic space or in gamma-transparent sources, the upper limit on diffuse neutrino flux $I_{v}(E)$ can be derived from electro-magnetic cascade radiation (Berezinsky \& Smirnov 1975). However, there can be the "hidden" neutrino sources where accompanying X-ray and gamma-radiations are absorbed. There are several known models of the hidden HE neutrino sources: The Young SN shells (Berezinsky \& Prilutsky 1987), opaque for all radiation except of neutrino during a time interval $t_{v} \sim 10^{3}-10^{4} \mathrm{~s}$. The Thorne-Zytkow star (Thorne \& Zytkow 1977), which is a neutron star submerged into a red giant. The cocooned massive black hole in AGN (Berezinsky \& Ginzburg 1981) where the electromagnetic radiation is absorbed in a cocoon around the black hole. The AGN with a standing shock in the vicinity of MBH (Stecker et al. 1991) can produce large flux of HE neutrinos accompanied by the relatively small flux of $\mathrm{X}$-ray radiation.

Recently we proposed the model for a powerful but shortlived hidden neutrino source which originates in a distant galactic nucleus prior to its collapse into the $\mathrm{MBH}$ (Berezinsky \& Dokuchaev 2001). In this model we assume that MBH is formed by the natural dynamical evolution of the central stellar cluster in the normal galactic nucleus. Dynamical evolution of dense central stellar clusters in the galactic nuclei is accompanied by the growth of a central gravitational potential $\phi \sim v^{2}$, where $v$ is a velocity dispersion of constituent stars. This evolution process is accompanied by the contraction of a central part of the galactic nucleus and terminated by formation of $\mathrm{MBH}$ (see for a review Rees 1984, and references therein). During this evolution a dense galactic nucleus inevitably proceeds through the stellar collision phase (Spitzer \& Saslaw 1966; Spitzer 1971; Rees 1984; Dokuchaev 1991), when normal stars in the cluster are destroyed in mutual collisions. A direct collision of two stars ends with their complete destruction if their relative kinetic energy exceeds the gravitational bound energy of stars. This condition is realized in stellar clusters with a high enough velocity dispersion. Namely, $v \geq v_{\text {esc }}=\left(2 G m_{*} / r_{*}\right)^{1 / 2}$ is fulfilled, where $v_{\text {esc }}$ is an escape velocity from the surface of star with mass $m_{*}$ and radius $r_{*}$. For a solar type star $v_{\mathrm{esc}} \simeq 620 \mathrm{~km} \mathrm{~s}^{-1}$. In a stellar cluster with $v>v_{\text {esc }}$ the normal stars are eventually destroyed in mutual collisions or in collisions with the extremely compact stellar remnants: neutron stars (NSs) or stellar mass black holes. Only compact stellar remnants survive through the normal star-destruction stage of evolution at $v \simeq v_{\text {esc }}$ and form the 
self-gravitating subsystem surrounded by a massive gas envelope. We shall refer to all compact stellar remnants as the NSs for brevity. The fireballs after numerous NS collisions in the contracting NS cluster result in the formation of a rarefied cavity in the massive gas envelope. Particles accelerated in this rarefied cavity interact with a dense envelope and produce secondary HE neutrinos. The accompanying gamma-radiation is fully absorbed in the case of thick enough envelope. The resulting hidden HE neutrino source is short-lived and very powerful: neutrino luminosity may exceed the Eddington limit for the electromagnetic radiation.

Berezinsky \& Dokuchaev (2001) considered the case when a self-gravitating cluster of NSs in the evolving galactic nucleus is formed simultaneously with a formation of massive gas envelope. It is possible because the time of collision of selfdestruction for normal stars appears to be of the same order as the time of NS cluster formation at a star-destruction stage of evolution of the galactic nucleus (see Sect. 3 for more details). In this case the newly formed NS cluster evolves much faster than a surrounding massive envelope. The corresponding parameters of massive envelope are fixed at the moment of NS cluster formation when $v \simeq v_{\text {esc }}$. Here we will consider another and probably more natural case, when a self-destruction of normal stars and formation of a massive envelope in the evolving galactic nucleus proceeds faster than a formation of the self-gravitating subsystem of NSs in it. This case corresponds to the initial formation of a supermassive star (SMS) with the individual NSs submerged in it. Correspondingly, a formation of self-gravitating subsystem of NSs is delayed with respect to the formation of SMS, and the evolutionary contraction of SMS must be taken into account. We show that the hidden source of HE neutrino arises in this case also and even more powerful than in the first scenario (Berezinsky \& Dokuchaev 2001) of a simultaneous formation of a massive envelope and NS cluster in the evolving galactic nucleus.

\section{Supermassive star formation}

The SMS may be formed from the gas produced in the process of destructive collisions of stars in the evolved stellar cluster with a velocity dispersion $v \geq v_{\text {esc }}$. A characteristic time-scale for stellar cluster dynamical evolution is the (two-body) relaxation time

$t_{\mathrm{r}}=\left(\frac{2}{3}\right)^{1 / 2} \frac{v^{3}}{4 \pi G^{2} m^{2} n \Lambda} \simeq 4.6 \times 10^{8} N_{8}^{2}\left(\frac{v}{v_{\mathrm{esc}}}\right)^{-3} \mathrm{yr}$,

where $N=10^{8} N_{8}$ is the number of stars in the cluster, $\Lambda=$ $\ln (0.4 N)$ is the Coulomb logarithm, $v$ is a star velocity dispersion, $n$ is a star number density, $m \simeq M_{\odot}$ is a mass of constituent stars. The corresponding virial radius of the stellar cluster is $R=G N m / 2 v^{2}$. The first equality in (1) is valid for the local values of parameters, meanwhile, the second one is valid only for the mean (virial) parameters of a self-gravitating cluster. At $v>v_{\text {esc }}$, where $v_{\text {esc }}$ is an escape velocity from the surface of constituent star, the time-scale for self-destruction of normal stars in mutual collisions is $t_{\text {coll }}=\left(v_{\text {esc }} / v\right)^{4} \Lambda t_{\mathrm{r}}$ (e.g. Dokuchaev 1991). Numerical modelling of catastrophic stellar collisions has been performed by e.g. Benz \& Hills (1992); Lai et al. (1993). We choose $v \simeq v_{\text {esc }}$ as a characteristic threshold value for a complete destruction of two stars and final production of unbound gas cloud. As a result, the stellar cluster in the evolved galactic nucleus with $v \geq v_{\text {esc }}$ transforms finally into the SMS due to catastrophic stellar collisions. At $v \simeq v_{\text {esc }}$ the time-scale for the formation of SMS due to destructive collisions of stars is of the same order as the relaxation time, $t_{\text {coll }}\left(v_{\text {esc }}\right) \sim t_{\mathrm{r}}\left(v_{\mathrm{esc}}\right)$. A total mass of the gas produced by destruction of normal stars composes the major part of a progenitor central stellar cluster in the galactic nucleus. Thus, the natural range of masses for the formed SMS is $M_{\mathrm{SMS}}=10^{7}-10^{8} M_{\odot}$.

A newly formed SMS with mass $M_{\mathrm{SMS}}$ and radius $R_{\mathrm{SMS}}$ gradually contracts due to radiation with the Eddington luminosity $L_{\mathrm{E}}=4 \pi G M_{\mathrm{SMS}} m_{\mathrm{p}} c / \sigma_{\mathrm{T}}$, where $m_{\mathrm{p}}$ is the proton mass and $\sigma_{\mathrm{T}}$ is the Thompson cross-section. A nonrotating SMS is a short-lived object that collapses due to post-Newtonian instability. Rotation provides the stabilization of SMS if the rotation energy is an appreciable part of its total energy $E_{\mathrm{SMS}} \simeq$ $\left(G M_{\mathrm{SMS}}^{2} / 2 R_{\mathrm{SMS}}\right)$. In general an evolution time of SMS is the Kelvin-Helmholtz time-scale $t_{\mathrm{SMS}}=E_{\mathrm{SMS}} / L_{\mathrm{E}} \propto R_{\mathrm{SMS}}^{-1}$. Stabilization of SMS by rotation (and additionally by internal magnetic field) ensures in principle its gradual contraction up to the gravitational radius (Zel'dovich \& Novikov 1971; Shapiro \& Teukolsky 1983; New \& Shapiro 2001). Thus, the maximum evolution time of SMS is of the order of the Eddington time $t_{\mathrm{E}}=0.1 M_{\mathrm{SMS}} c^{2} / L_{\mathrm{E}} \simeq 4.5 \times 10^{7} \mathrm{yr}$. We approximate the distribution of gas in a SMS by polytropic model with an adiabatic index $\gamma=4 / 3$. For this value of adiabatic index the central gas density in SMS is $\rho_{\mathrm{c}}=k_{\mathrm{c}} n_{\mathrm{SMS}} m_{\mathrm{p}}$ and central sound velocity $c_{\mathrm{s}, \mathrm{c}}=k_{\mathrm{s}} v_{\mathrm{SMS}}$, where $n_{\mathrm{SMS}}$ is a SMS mean number density, $v_{\mathrm{SMS}}=\left(G M_{\mathrm{SMS}} / 2 R_{\mathrm{SMS}}\right)^{1 / 2}$ is a SMS virial velocity and numerical constants $k_{\mathrm{c}} \simeq 54.2$ and $k_{\mathrm{s}} \simeq 1.51$ respectively.

\section{Neutron star cluster formation}

The compact stellar remnants in the form of NSs and stellar mass black holes submerged into the newly formed SMS. This is a specific feature of SMS formed in the evolving stellar cluster. Let SMS contains a small fraction of identical NSs with a total mass $M_{\mathrm{NS}}=f_{\mathrm{NS}} M_{\mathrm{SMS}}, f_{\mathrm{NS}} \ll 1$ and a typical mass of individual NS is $m_{\mathrm{NS}}=1.4 M_{\odot}$. In numerical estimations we will use $f_{\mathrm{NS}}=10^{-2} f_{-2}$ with $f_{-2} \sim 1$. An individual NS with a mass $m$ and local velocity $V$ is spiraling down the SMS center under influence of the dynamical friction force (Chandrasekhar 1943; Ostriker 1999) $F_{\mathrm{df}}=4 \pi I(G m)^{2} \rho_{\mathrm{SMS}} / V^{2}$, where $\rho_{\mathrm{SMS}}$ is a SMS gas density. The dimensionless factor here is $I \simeq 1$ in the case of $V \simeq c_{\mathrm{s}}$, where $c_{\mathrm{s}}$ is a sound velocity in SMS. Deep inside SMS the individual NSs are spiralling down and combine into a fast evolving self-gravitating subsystem (NS cluster). A corresponding time-scale for the NS dynamical friction drag toward the SMS center is $t_{\mathrm{df}}=V / \dot{V}=m V / F_{\mathrm{df}}$. At the moment of SMS formation, when $V \sim c_{\mathrm{s}} \sim v_{\mathrm{esc}}$, a time-scale of NS friction drag is of the same order as the time-scale of SMS formation, $t_{\mathrm{df}}\left(v_{\mathrm{esc}}\right) \sim t_{\text {coll }}\left(v_{\mathrm{esc}}\right) \sim t_{\mathrm{r}}\left(v_{\mathrm{esc}}\right)$. Thus, in general it is possible either (i) a simultaneous formation of a self-gravitating cluster of NSs and the host SMS or (ii) a delayed formation of a selfgravitating cluster of NSs inside the host SMS. The case (i) was considered by Berezinsky \& Dokuchaev (2001) and the case (ii) we describe below. In particular, in the case (ii) it must be taken into account a possibility of the SMS contraction prior to the formation of a central NS cluster.

The NS dynamical friction time-scale for a mean NS inside the host SMS is decreasing during contraction of SMS as $t_{\mathrm{df}} \propto R^{3 / 2}$. On the contrary, the SMS evolution time-scale is increasing with SMS contraction as $t_{\mathrm{SMS}} \propto R^{-1}$. Thus, the subsystem of NSs begins evolving faster than the host SMS after reaching the stage when $t_{\mathrm{df}} \simeq t_{\mathrm{SMS}}$. At this stage all NSs sink deep to a central part of SMS and form there a self-gravitating 
cluster. From relation $t_{\mathrm{df}}(R) \simeq t_{\mathrm{SMS}}(R)$ we find the corresponding SMS radius at the moment of NS cluster formation inside it:

$R_{\mathrm{SMS}} \simeq\left(\frac{9 I^{2}}{8 \pi^{2}} \frac{G m_{\mathrm{NS}}^{2} M_{\mathrm{SMS}} \sigma_{\mathrm{T}}^{2}}{c^{2} m_{\mathrm{p}}^{2}}\right)^{1 / 5} \simeq 4.6 \times 10^{15} M_{8}^{1 / 5} \mathrm{~cm}$,

where $M_{8}=M_{\mathrm{SMS}} /\left(10^{8} M_{\odot}\right)$. Respectively a mean gas density

$n_{\mathrm{SMS}} \simeq \frac{3}{4 \pi} \frac{M_{\mathrm{SMS}}}{R_{\mathrm{SMS}}^{3} m_{\mathrm{p}}} \simeq 2.9 \times 10^{17} M_{8}^{2 / 5} \mathrm{~cm}^{-3}$,

a mean column density

$X_{\mathrm{SMS}} \simeq n_{\mathrm{SMS}} m_{\mathrm{p}} R_{\mathrm{SMS}} \simeq 2.2 \times 10^{9} M_{8}^{3 / 5} \mathrm{~g} \mathrm{~cm}^{-2}$,

a virial velocity $v_{\mathrm{SMS}}=\left(G M_{\mathrm{SMS}} / 2 R_{\mathrm{SMS}}\right)^{1 / 2} \simeq 0.04 M_{8}^{2 / 5} c$, a SMS evolution time $t_{\text {SMS }}=t_{\text {SMS }}\left(R_{\text {SMS }}\right) \simeq 7.3 \times 10^{5} M_{8}^{4 / 5} \mathrm{yr}$ and a surface black-body temperature $T_{\mathrm{SMS}} \simeq 3 \times 10^{4} M_{8}^{3 / 20} \mathrm{~K}$. Note that the only free parameter in these relations is $M_{\mathrm{SMS}}$. A self-gravitating NS cluster with a radius $R_{\mathrm{NS}} \ll R_{\mathrm{SMS}}$ is formed when a total mass of NSs inside this radius becomes of order of an ambient gas mass, $M_{\mathrm{NS}} \simeq(4 \pi / 3) R_{\mathrm{NS}}^{3} \rho_{\mathrm{c}}$, where $\rho_{\mathrm{c}}$ is a central SMS density. From this relation the initial radius of NS cluster is $R_{\mathrm{NS}}=f_{\mathrm{NS}}^{1 / 3} k_{\mathrm{c}}^{-1 / 3} R_{\mathrm{SMS}}$ and velocity dispersion $v_{\mathrm{NS}}=f_{\mathrm{NS}}^{1 / 3} k_{\mathrm{c}}^{1 / 3} v_{\mathrm{SMS}}$, respectively.

\section{Neutron star cluster evolution}

The formed NS cluster evolves much more faster than a host SMS and collapses finally into the MBH. A suitable approximation for the dynamical evolution of NS cluster in our case is the homologous "evaporation" model (e.g. Spitzer 1987; Saslaw 1987). This model describes the two-body interactions of stars in the cluster with an assumption that a fast star escapes (evaporates) from the cluster with a zero total energy. Accordingly, a total virial energy of the cluster remains constant $E=-N m v^{2} / 2=$ const. during evaporation of fast stars. The velocity dispersion in the evolving cluster is growing as $v \propto N^{-1 / 2}$ and cluster radius diminishes as $R \propto N^{2}$ with a diminishing of number $N$ of remaining stars in the cluster. After reaching $v \simeq 0.3 c$, which is onset of the NS cluster global dynamical instability, the remaining NS cluster collapses to the MBH (e.g. Zel'dovich \& Poduretz 1965; Quinlan \& Shapiro 1987). The rate of NS evaporation from the cluster is

$\dot{N}_{\mathrm{ev}} \simeq \alpha N t_{\mathrm{r}}^{-1}$,

where a relaxation time $t_{\mathrm{r}}$ is given by (1) and constant $\alpha \sim$ $10^{-3}-10^{-2}$ according to the numerical calculations in the Fokker-Plank approximation (Spitzer 1987; Saslaw 1987; Cohn 1980). Integration of (5) together with the relation $E=$ const. results in the nondissipative evolution due to evaporation of fast stars:

$N(t)=N_{\mathrm{NS}}\left(1-\frac{t}{t_{\mathrm{ev}}}\right)^{2 / 7}$,

where the cluster evolution time $t_{\mathrm{ev}}=a_{\mathrm{ev}} t_{\mathrm{r}}$, numerical constant $a_{\mathrm{ev}}=(2 / 7) \alpha^{-1}$ and $t_{\mathrm{r}}$ is the relaxation time at the moment of NS cluster formation $t=0$. In numerical estimations we put $a_{\mathrm{ev}}=10^{2} a_{2}$ with $a_{2} \sim 1$.

The random collisions of NSs and their evaporation from the cluster occur at the same time. A rate of NS collisions in the cluster with the gravitational radiation losses taken into account (Quinlan \& Shapiro 1987; Dokuchaev et al. 1998) is

$\dot{N}_{\text {cap }} \simeq 36 \sqrt{2}\left(\frac{v}{c}\right)^{31 / 7} \frac{1}{N} \frac{c}{r_{\mathrm{g}}}$

where $r_{\mathrm{g}}=2 G m_{\mathrm{NS}} / c^{2}$ is a gravitational radius of NS. According to Eq. (6) the collision rate (7) is gradually growing due to the evaporative dynamical evolution of cluster as $\dot{N}_{\text {cap }} \propto v^{45 / 7}$. The dissipative process of collisions is negligible for the dynamical evolution of a cluster in comparison with the nondissipative evaporation until $\dot{N}_{\text {cap }}<\dot{N}_{\text {ev }}$. This inequality is equivalent to the condition $v<v_{\text {cap }}$, where

$v_{\text {cap }}=\left(\frac{4}{7 \sqrt{3}} \frac{\Lambda}{a_{\mathrm{ev}}}\right)^{7 / 10} c \simeq 8.7 \times 10^{-2} a_{2}^{-7 / 10} c$.

At $v>v_{\text {cap }}$ the NS collisions becomes a major evolution process in cluster. The contraction of cluster is accelerated and it finally collapses to $\mathrm{MBH}$.

\section{Fireballs and rarefied cavity inside the SMS}

We assume that each NS collision is accompanied by the generation of relativistic fireball with a total energy $E_{0}=10^{52} E_{52} \mathrm{erg}$, where $E_{52} \sim 1$. The physics of fireballs is extensively elaborated in the recent years for the gamma-ray burst (GRB) models (for review see e.g. Piran 2000, and references therein).

Although in our case at least the first fireball is born in SMS with large density $n_{\text {SMS }}$ given by Eq. (3), the dynamics of the fireballs remains the same as in the standard theory. Consider the first fireball assuming it to be spherically symmetric, which in fact is not a necessary assumption.

The tremendous energy release $E_{0} \sim 10^{52}$ erg occurs within the small volume with radius $R_{i} \sim 10^{7} \mathrm{~cm}$ and the radiation pressure $p_{i} \sim E_{0} / R_{i}^{3}$ by many orders of magnitude exceeds the external pressure $p \sim n_{\mathrm{SMS}} k T$. The pressure in the fireball, which consists of relativistic $\gamma \mathrm{e}^{+} \mathrm{e}^{-}$gas with small admixture of the baryons, accelerates fireball to large Lorentz-factor $\Gamma_{\mathrm{f}}$. The relativistic shock, with Lorentz factor $\Gamma_{\mathrm{sh}}=\sqrt{2} \Gamma_{\mathrm{f}}$ propagates ahead of the fireball pushing the SMS gas. The shock becomes nonrelativistic at the Sedov length

$l_{\mathrm{S}}=\left(3 E_{0} / 4 \pi n_{\mathrm{SMS}} m_{\mathrm{H}} c^{2}\right)^{1 / 3}=1.7 \times 10^{12} \mathrm{~cm}$.

This value gives an order of magnitude estimate for cavity radius (see below).

The second fireball propagates in the rarefied cavity produced by the first fireball, and it overtakes the first one at about Sedov length, when the first shock is non-relativistic. The shocks from the first and successive fireballs merge into one, hence the following scenario is plausible.

The numerous fireballs after successive NS collisions in a dense cluster provide the central energy source in our model. The power of this source is not limited by the Eddington luminosity in contrast to the accretion sources. The radius of NS cluster is very small in comparison with the host SMS radius $R_{\mathrm{SMS}}$. The shocks produced by numerous fireballs dig out a rarefied cavity with a small radius in comparison with $R_{\mathrm{SMS}}$. Consider the merging shock in more details.

The early nonstationary stage of shock expansion can be described by the self-similar spherical shock solution for a central source with energy varying in time as $E=A t^{k}$ with $A=$ const. and $k=$ const. (Ostriker $\&$ McKee 1995; Dokuchaev 2002). The 
particular case of $k=0$ corresponds to the Sedov instant shock solution (see e.g. Landau \& Lifshitz 1959). Meanwhile the nonstationary shock from multiple fireballs corresponds to a permanent energy injection into the shock, or injection shock with a central source of constant luminosity, $k=1, L=A, E=L t$. The radius of a self-similar expanding shock grows with time as

$R=R(t)=\beta\left(\frac{A}{\rho_{\mathrm{c}}}\right)^{1 / 5} t^{(2+k) / 5}$,

where numerical constant $\beta=\beta(\gamma, k)$ depends on the gas adiabatic index $\gamma$ and $k$, e.g. $\beta(4 / 3,1)=0.793$ (Dokuchaev 2002). The velocity of shock expansion is

$u=\frac{\mathrm{d} R}{\mathrm{~d} t}=\frac{(2+k) R}{5 t}$

The maximum radius of the expanding strong shock $R_{\mathrm{sh}}$ is obtained from the equality $u\left(R_{\mathrm{sh}}\right)=c_{\mathrm{s}, \mathrm{c}}$ by using (10) and (11):

$R_{\mathrm{sh}}=\left[\left(\frac{2+k}{5 c_{\mathrm{s}, \mathrm{c}}}\right)^{2+k} \beta^{5} \frac{A}{\rho_{\mathrm{c}}}\right]^{1 /(3-k)}$.

Respectively the maximum time of a strong shock expansion is $t_{\mathrm{sh}}=[(2+k) / 5]\left(R_{\mathrm{sh}} / c_{\mathrm{s}, \mathrm{c}}\right)$. The expansion law for a $k=1$ injection shock corresponds to constant energy flux (or constant source luminosity) carried by the swept-out gas. This constant luminosity shock solution is applicable only to the early nonstationary stage of cavity formation at $t<t_{\mathrm{sh}}$.

At the late stationary stage at $t \geq t_{\mathrm{sh}}$ the boundary of the cavity is supported in a dynamic equilibrium by the relativistic wind from successive fireballs. The radius of a stationary cavity $R_{\text {cav }}$ is determined from the energy flux balance on its boundary. The central source power or luminosity is $L=\dot{N}_{\text {cap }} E_{0}$, where $\dot{N}_{\text {cap }}$ is from (7). After formation of cavity the energy generated in the central source is carried out by a relativistic wind. Just outside $R_{\text {cav }}$ the energy flux is carried by a hydrodynamic flow $L=4 \pi R_{\mathrm{cav}}^{2} \rho v\left(w+v^{2} / 2\right)$ with a gas velocity $v \sim c_{\mathrm{s}, \mathrm{c}}$, where $c_{\mathrm{s}, \mathrm{c}}$ and $\rho_{\mathrm{c}}$ are respectively a sound velocity and gas density in the central part of SMS. The gas produces some work under expansion, so the energy flux $L$ contains an enthalpy density $w=\varepsilon+p / \rho=c_{\mathrm{s}}^{2} /(\gamma-1)$, where $\varepsilon=c_{\mathrm{s}}^{2} /[\gamma(\gamma-1)], p, \rho$ and $\gamma$ are respectively a gas internal energy density, pressure, density and adiabatic index. From the energy flux balance relation we determine a stationary cavity radius for $\gamma=4 / 3$

$R_{\mathrm{cav}}=\left(\frac{\dot{N}_{\mathrm{cap}} E_{0}}{14 \pi \rho_{\mathrm{c}} c_{\mathrm{s}, \mathrm{c}}^{3}}\right)^{1 / 2}$.

For the stationary cavity existence the time interval between successive fireballs $t_{\text {cap }}=\dot{N}_{\text {cap }}^{-1}$ must be less than that for cavity shrinking (or spreading) $t_{\mathrm{cav}}=R_{\mathrm{cav}} / c_{\mathrm{s}, \mathrm{c}}$. This requirement, $t_{\text {cap }}<t_{\text {cav }}$, with a help of (7) and (13) can be written as condition $v>v_{\text {cav }}$, where

$v_{\text {cav }} \simeq 7.3 \times 10^{-2} f_{-2}^{7 / 27} E_{52}^{-7 / 135} M_{8}^{91 / 225} c$,

with numerical values $v_{\text {cav }} \simeq 4.3 v_{\mathrm{NS}}$. The corresponding minimal radius of a stationary cavity $R_{\min }=R_{\text {cav }}\left(v_{\text {cav }}\right)$ is

$R_{\min } \simeq\left(\frac{E_{0}}{4 \pi \rho_{\mathrm{c}} c_{\mathrm{s}, \mathrm{c}}^{2}}\right)^{1 / 3} \simeq 2.1 \times 10^{12} E_{52}^{1 / 3} M_{8}^{-2 / 5} \mathrm{~cm}$.

It follows from (15) that $R_{\min }$ is independent of $f_{\mathrm{NS}}$. At the moment of a stationary cavity formation inside SMS, $v=v_{\text {cav }}$, we have respectively a radius of the NS cluster $R\left(v_{\text {cav }}\right) \simeq$ $7.5 \times 10^{11} f_{-2}^{17 / 27} E_{52}^{28 / 135} M_{8}^{41 / 225} \mathrm{~cm}$, a number of NSs in the cluster at the moment of cavity formation $N\left(v_{\text {cav }}\right) \simeq 3.8 \times$ $10^{4} f_{-2}^{31 / 27} E_{52}^{14 / 135} M_{8}^{223 / 225}$, a NS cluster evolution time $t_{\mathrm{ev}}\left(v_{\mathrm{cav}}\right) \simeq$ $0.3 a_{2} f_{-2}^{41 / 27} E_{52}^{49 / 135} M_{8}^{173 / 225}$ yr and central source luminosity inside a SMS $L\left(v_{\text {cav }}\right) \simeq 8.6 \times 10^{48} E_{52}^{2 / 3} M_{8}^{4 / 5} \mathrm{erg} / \mathrm{s}$. After the formation of a stationary cavity inside the SMS we have a hierarchy of radii, $R\left(v_{\text {cav }}\right)<R_{\min } \ll R_{\mathrm{SMS}}$, which justifies the using of central values for a gas density $\rho_{\mathrm{c}}$ and sound velocity $v_{\mathrm{s}, \mathrm{c}}$.

The central source power and cavity radius are gradually growing due to the evolutionary grows of NS collision rate. The rate of NS collisions and the power of the central source reach the maximum value at $v \simeq v_{\text {cap }}$ from (8). The corresponding maximum central source power $L_{\max }=\dot{N}_{\text {cap }}\left(v_{\text {cap }}\right) E_{0}$ is

$L_{\max } \simeq 2.8 \times 10^{49} a_{2}^{-9 / 2} f_{-2}^{-5 / 3} E_{52} M_{8}^{-9 / 5} \mathrm{erg} \mathrm{s}^{-1}$.

By substituting this luminosity in (13) we find that the maximal cavity radius $R_{\max }=R_{\text {cav }}\left(v_{\text {cap }}\right)$ is

$R_{\max } \simeq 3.8 \times 10^{12} a_{2}^{-9 / 4} f_{-2}^{-5 / 6} E_{52}^{1 / 2} M_{8}^{-17 / 10} \mathrm{~cm}$.

Respectively at $v=v_{\text {cap }}$ the NS cluster radius $R\left(v_{\text {cap }}\right) \simeq 3.6 \times$ $10^{11} a_{2}^{14 / 5} f_{-2}^{5 / 3} M_{8}^{9 / 5} \mathrm{~cm}$, number of NS in the cluster $N\left(v_{\text {cap }}\right) \simeq$ $2.7 \times 10^{4} a_{2}^{7 / 5} f_{-2}^{5 / 3} M_{8}^{9 / 5}$ and NS cluster evolution time $t_{\mathrm{ev}}\left(v_{\mathrm{cap}}\right) \simeq$ $31.3 a_{2}^{59 / 10} f_{-2}^{10 / 3} M_{8}^{18 / 5}$ days.

Collisions of NSs during a cluster lifetime $t_{\mathrm{ev}}$ at $v=$ $v_{\text {cap }}$ supply the total energy $\mathcal{E}_{\max } \simeq L_{\max } t_{\mathrm{ev}} \simeq 7.6 \times$ $10^{55} a_{2}^{7 / 5} f_{-2}^{5 / 3} E_{0} M_{8}^{9 / 5}$ erg into the cavity. This energy is far less than a SMS binding energy $E_{\mathrm{SMS}} \simeq G M_{\mathrm{SMS}}^{2} / 2 R_{\mathrm{SMS}} \simeq 2.9 \times$ $10^{59} M_{8}^{9 / 5} \mathrm{erg}$, and so the shocks after numerous fireballs do not influence the SMS internal structure.

\section{Production and detection of high-energy neutrinos}

We shall study here the acceleration of protons by relativistic fireballs produced by NS collisions and the high-energy neutrino radiation produced in $p \gamma$ and $p p$ collisions. We assume a standard GRB fireball which propagates at the baryon-dominated stage with the Lorentz-factor $\Gamma=10^{2} \Gamma_{2}, \Gamma_{2} \sim 1$ and carries a baryonic mass $M_{0}=E_{0} / \Gamma c^{2} \simeq 5.6 \times 10^{-5} E_{52} \Gamma_{2}^{-1} M_{\odot}$.

The acceleration occurs due to internal shocks inside the fireball, Waxman (1995), and due to external shocks, Vietri (1995). We shall consider first the internal shock acceleration and highenergy neutrino production following Waxman $(1995,2001)$. This scenario we consider as the basic one for a prediction of neutrino flux in our model.

The standard explanation of the observed GRBs, Piran (2000), is given under an assumption that fireball consists of the mildly relativistic sub-shells. The inner sub-shells are moving faster than the outer ones, and their collisions produce mildly relativistic shocks. The protons are accelerated by these shocks with the standard spectrum $\propto E^{-2}$ and with a maximum acceleration energy $E_{\max } \sim 1 \times 10^{21} \mathrm{eV}$, Waxman (1995). The specific feature of our case is a relatively short time of fireball existence in the cavity, $t \sim R_{\text {cav }} / c$. This time, however, is long enough for acceleration of protons up to the maximum energy $E_{\max } \sim 10^{21} \mathrm{eV}$. In the rest-frame $\mathcal{K}^{\prime}$ the acceleration time is $t_{\mathrm{a}}^{\prime} \sim r_{\mathrm{L}}^{\prime} / c$, where $r_{\mathrm{L}}^{\prime}$ is the Larmor radius. Following to 
Piran (2000), an equipartition magnetic field $B_{\text {eq }}^{\prime}$ in the relativistic wind can be estimated as

$$
\frac{B_{\mathrm{eq}}^{\prime 2}}{8 \pi} \sim \xi_{\mathrm{B}} \omega_{\gamma}^{\prime} \sim 2 \xi_{\mathrm{B}} \frac{L_{\gamma}}{\Gamma^{2} r^{2} c},
$$

where unprimed symbols correspond to quantities in the laboratory frame, $\omega_{\gamma}^{\prime}$ is photon energy density, $\xi_{\mathrm{B}} \sim 1$ is an equipartition parameter and $L$ is a fireball luminosity. Using $L_{\gamma} \sim$ $10^{52} \mathrm{erg} / \mathrm{s}$ and $r \sim R_{\text {cav }}$ we obtain the acceleration time as $t_{\mathrm{a}} \sim 50 \mathrm{~s}$ for particles with $E_{\max } \sim 10^{21} \mathrm{eV}$. With an equipartition magnetic field (18) the maximum acceleration energy is $E \sim e B_{\text {eq }}^{\prime} R_{\text {cav }} \sim 10^{21} \mathrm{eV}$ in agreement with the Waxman (1995) model.

High-energy neutrinos are produced in $p \gamma$ collisions inside the fireball by the Waxman \& Bahcall (1997) mechanism. Due to threshold of pion production in $p \gamma$ collisions the neutrino flux has a low-energy suppression. The low-energy part of neutrino spectrum is produced in $p p$ collisions of accelerated protons with a gas inside the cavity and in the SMS interiors. Reflecting from the fireball, the cavity gas penetrates into the fireball at the Larmor radius distance $d \sim r_{\mathrm{L}}$ for a particle with energy $E^{\prime} \sim \Gamma m_{\mathrm{p}}$. In this region accelerated protons interact with an accumulated gas, producing pions and neutrinos. However, below we shall consider more efficient mechanisms for production of low-energy neutrinos.

We assume, as it is usually used in the GRB calculations, the equipartition of total energy distribution in the form of UHE protons $W_{\mathrm{p}}$, neutrinos $W_{v}$, GRB photons $W_{\mathrm{GRB}}$ and the energy transferred to the shock in SMS. Then for neutrino luminosity during the maximum activity of the considered central source we have $L_{v} \sim(1 / 4) L_{\max }$ with $L_{\max }$ given by (16). Though neutrino spectrum is presented by two components, for simplicity of further estimates we assume the universal spectrum $\propto 1 / E^{2}$. Normalizing this spectrum to $L_{v}$, we obtain for the neutrino generation rate

$$
Q_{v}=\frac{1}{4} \frac{L_{\max }}{E^{2} \ln \left(E_{v, \max } / E_{v, \min }\right)} .
$$

Taking into account the neutrino oscillation, one obtains $v_{\mu}+\bar{v}_{\mu}$ neutrino flux at a distance $r$ from the source $F_{v_{\mu}+\bar{v}_{\mu}}(E) \stackrel{=}{=}$ $(1 / 3) Q_{v}(E) / 4 \pi r^{2}$.

We shall discuss here only the detection of $\mathrm{TeV}$ neutrinos from the considered hidden source by a future $1 \mathrm{~km}^{2}$ ice or underwater detector. The fluxes of UHE neutrinos up $10^{19} \mathrm{eV}$ are most probably also detectable, but they need the knowledge of the details of neutrino detection. This task is beyond the scope of this paper.

Crossing the Earth, neutrinos with energies $E>0.1-1 \mathrm{TeV}$ produce deep underground an equilibrium flux of HE muons, which can be calculated (Berezinsky 1990) as

$F_{\mu}(>E)=\frac{\sigma_{0} N_{\mathrm{A}}}{b_{\mu}} Y_{\mu}\left(E_{\mu}\right) \frac{L_{\max }}{12 \zeta E_{\mu}} \frac{1}{4 \pi r^{2}}$,

where $\sigma_{0}=1 \times 10^{-34} \mathrm{~cm}^{2}$ is the normalization cross-section, $N_{\mathrm{A}}=6 \times 10^{23}$ is the Avogadro number, $b_{\mu}=4 \times 10^{-6} \mathrm{~cm}^{2} / \mathrm{g}$ is the rate of muon energy losses, $\zeta=\ln E_{\max } / E_{\min }$, and $Y_{\mu}(E)$ is the integral muon moment of $v_{\mu} N$ interaction (Berezinsky et al. 1990; Berezinsky 1990). The most effective energy of muon detection is $E_{\mu} \geq 0.1-1 \mathrm{TeV}$, because these muons cross the large part of $1 \mathrm{~km}^{3}$ detector, and their directions can be reliably measured. The number of muons $N\left(v_{\mu}\right)=F_{\mu} S t_{\mathrm{ev}}$ with $E_{\mu} \geq 1 \mathrm{TeV}$ detected during the burst duration $t_{\mathrm{ev}}=31.3$ days by the underwater detector with an effective area $S$ at distance $r$ from the source is given by

$$
N\left(v_{\mu}\right) \simeq 56 \frac{L_{\max }}{2.8 \times 10^{49} \mathrm{erg} \mathrm{s}^{-1}} \frac{S}{1 \mathrm{~km}^{2}}\left(\frac{r}{10^{3} \mathrm{Mpc}}\right)^{-2} .
$$

The detected muons with $E_{\mu} \geq 1 \mathrm{TeV}$ show the direction to the source with an accuracy $2^{\circ}-3^{\circ}$.

The other acceleration mechanism due to the external ultrarelativistic shocks, Vietri (1995), provides mostly $p p$ neutrino flux with energies below $1 \mathrm{TeV}$. Consider the shock which is produced when a fireball hits the inner surface of SMS. The shock propagates in ultra-relativistic regime at distance of order of the Sedov length, before it overtakes the slow main shock and merges with it. The gas density in this region rises from that of the cavity $n_{\mathrm{c}} \sim 4 \times 10^{11} \mathrm{~cm}^{-3}$ to maximum density $n_{\max } \simeq$ $54.2 n_{\mathrm{SMS}}$, with $n_{\mathrm{SMS}} \simeq 3 \times 10^{17} \mathrm{~cm}^{-3}$ given by (3). The highenergy proton initiates a nuclear cascade due to pion production. The energy dissipates from the cascade through cascade-pion decays. The decays of neutral pions initiate the electro-magnetic cascade and the released energy contributes to the shock and heating of SMS. The column density of SMS, $X_{\mathrm{SMS}} \simeq 2.3 \times$ $10^{9} M_{8}^{3 / 5} \mathrm{~g} \mathrm{~cm}^{-2}$, is large enough to absorb all produced particles except the secondary neutrinos. Neutrinos are produced in decays of the charged pions. The critical Lorentz factor of the decaying charged pion is determined by condition $\Gamma_{\mathrm{c}} \sim$ $\left(\sigma_{\pi N} \bar{n} \tau_{\pi} c\right)^{-1} \sim 5 \times 10^{4}$, where $\sigma_{\pi N} \simeq 25 \mathrm{mb}$ is $\pi N$ cross-section and $\tau_{\pi}=2.6 \times 10^{-8} \mathrm{~s}$ is the charged pion lifetime, and for the mean gas density we used $\bar{n} \sim 1 \times 10^{18} \mathrm{~cm}^{-3}$, larger than $n_{\text {SMS }}$ and smaller than $n_{\max }$. As a result we obtain for the maximum energy of the secondary neutrinos $E_{v, \text { max }} \sim 1 \mathrm{TeV}$.

Acceleration by ultra-relativistic shock at the inner boundary of SMS proceeds in a specific way because of the large density of matter there and large magnetic field. Consider one of the fireballs hitting the inner boundary of SMS. Propagating through the dense gas the shock, running ahead of the fireball, accelerates the protons. A proton from the SSM interior (upstream region), which makes its first downstream - upstream - downstream (d-u-d) cycle, i.e. crossing the shock and scattering upstream back to downstream, increases its energy by a factor $\Gamma_{\text {sh }}^{2}$. In the consequent d-u-d cycles the energy gain is only about 2 , Gallant \& Achterberg (1999), (see also Lemoine \& Revenu (2005) for a recent work). The maximum acceleration energy is determined by the number of completed d-u-d cycles and is given by $t_{\mathrm{a}} \sim t_{\text {int }}$ condition, where $t_{\mathrm{a}}$ and $t_{\text {int }}$ are acceleration and interaction time, respectively. They are given by $t_{\mathrm{a}} \sim E / e c B \Gamma_{\mathrm{sh}}$, Gallant $\&$ Achterberg (1999), and $t_{\text {int }} \sim 1 / c \sigma_{p p} n_{\text {SMS }}$. Acceleration time is short because of very large magnetic field accumulated at the inner boundary of the shock. The magnetic field in a fireball, $B_{\text {eq }}$, given by Eq. (18) reaches $4 \times 10^{6} \mathrm{G}$, when it hits the SMS. The each successive fireball adds the magnetic flux, and though not being summed up coherently, magnetic field increases. For $B \sim$ $4 \times 10^{6} \mathrm{G}$ the maximum energy $E_{\max } \sim 1 \times 10^{17} \mathrm{eV}$. Fortunately, this energy, being unreliably estimated, is not important for our calculations of neutrino flux, because all high-energy protons loose energy by producing nuclear-electromagnetic cascade.

The proton with an energy $E_{\mathrm{p}}$ transfers fraction of energy $f\left(E_{\mathrm{p}}\right)$ to neutrinos through decays of cascading charged pions, $\Sigma E_{v}=f\left(E_{\mathrm{p}}\right) E_{\mathrm{p}}$. The fraction $f=1 / 2$ at $E_{\mathrm{p}} \leq 10^{13} \mathrm{eV}$ and correspondingly $f \sim 0.05$ at $E_{\mathrm{p}} \sim 10^{17} \mathrm{eV}$, because in the cascading process all neutral pions decay. For the total energies of protons and neutrinos $W_{v}=\bar{f} W_{\mathrm{p}}$, where $\bar{f}$ is the value averaged over spectrum of accelerated protons. We will assume $\bar{f} \sim 0.1$. 
It is easy to see that neutrino signal in the detector is determined by $W_{v}$. It occurs because the cross-section $v N$ at the energies of interest is $\sigma(E)=\sigma_{0} E$, with $\sigma_{0}=0.8 \times 10^{-38} \mathrm{~cm}^{2} / \mathrm{GeV}$. The total number of neutrino events in $1 \mathrm{~km}^{3}$ detector is given by

$N_{\text {tot }}=\frac{N_{\mathrm{N}} \sigma_{0}}{4 \pi r^{2}} \int N_{v}(E) E \mathrm{~d} E=\frac{N_{\mathrm{N}} \sigma_{0} W_{v}}{4 \pi r^{2}}$,

where the number of target nucleons is $N_{\mathrm{N}}=6 \times 10^{38}$ and $W_{v} \sim$ $0.1 W_{\mathrm{p}}$. For $r=1 \mathrm{Gpc}$ and $W_{\mathrm{p}}=(1 / 4) W_{\mathrm{tot}}=1.9 \times 10^{55} \mathrm{erg}$, it results in $N_{\text {tot }}=47$ of low-energy $\left(E_{v}<1 \mathrm{TeV}\right)$ neutrino events in $1 \mathrm{~km}^{3}$ detector. The neutrinos produced in the SMS by high-energy protons, accelerated in the inner shocks, double this number of neutrino events.

On may wonder about the influence of adiabatic energy losses of accelerated protons on neutrino fluxes, estimated above. The role of adiabatic energy losses has been critically discussed by Rachen \& Meszaros (1998) (see also reply by Waxman (2004). For the calculations above, the adiabatic energy losses are irrelevant, because in all cases neutrinos are produced in situ with the acceleration of particles.

How many these hidden sources are expected? A further (after neutrino burst) evolution of SMS with a mass $M \sim 10^{8} M_{\odot}$ inevitably results in formation of a massive black hole and the AGN activity. Considering the described hidden source as a "pre-AGN" stage one can estimate an expected number of hidden sources inside the cosmological horizon. Since a hidden source and AGN are considered as the different stages of the same evolving galactic nucleus, the probability to have a hidden source in the set of AGN is given by the ratio of durations of the corresponding evolution phases, $t_{\mathrm{ev}} / t_{\mathrm{AGN}}$, where $t_{\mathrm{AGN}}$ is a duration of AGN phase. Then the number of hidden sources within the horizon is given by

$N_{\mathrm{HS}} \sim \frac{4}{3} \pi\left(3 c t_{0}\right)^{3} n_{\mathrm{AGN}} t_{\mathrm{s}} / t_{\mathrm{AGN}}$,

where $(4 \pi / 3)\left(3 c t_{0}\right)^{3}$ is the cosmological volume inside the horizon $c t_{0}$ and $n_{\mathrm{AGN}}$ is the number density of AGN. The estimates for $n_{\mathrm{AGN}}$ and $t_{\mathrm{AGN}}$ taken for different populations of AGN results in $N_{\mathrm{HS}} \sim 1-10$.

Can the hidden sources be observed in electromagnetic radiations? X-ray and gamma-ray radiations are fully absorbed due to tremendous thickness of SMS, $X_{\text {SMS }} \sim 2 \times 10^{9} \mathrm{~g} \mathrm{~cm}^{-2}$. However the thermaized radiation can propagate diffusively and reach the the outer surface of SMS. This process has been considered in Berezinsky \& Dokuchaev (2001) for the case of a massive shell. In case of SMS, the diffusion is much slower and the thermalized radiation reach the outer surface much later, and thus will be emitted with a lower luminosity. The diffusion coefficient $D \sim c l_{\mathrm{d}}$, where a diffusion length is $l_{\mathrm{d}}=1 /\left(\sigma_{\mathrm{T}} n_{\mathrm{SMS}}\right)$, and $\sigma_{\mathrm{T}}$ is the Thompson cross-section. A mean time of the radiation diffusion through SMS is very long, $t_{\mathrm{d}} \sim R_{\mathrm{SMS}}^{2} / D \sim 4 \times$ $10^{6} M_{8}^{4 / 5} \mathrm{yr}$, in comparison with a lifetime of the NS cluster inside the SMS. Since the dispersion of the diffusion time distribution $\sigma \sim t_{\mathrm{d}}$, the surface luminosity of the central source is $L_{\mathrm{bb}} \sim E_{\mathrm{tot}} / \sigma \sim L_{\mathrm{max}} t_{\mathrm{ev}} / t_{\mathrm{d}} \sim 5 \times 10^{41} \mathrm{erg} / \mathrm{s}$ and hence is negligible in comparison with the Eddington luminosity of the host SMS.

\section{Conclusions}

In this paper we studied the natural scenario for massive black hole formation in the process of dynamical evolution of a compact stellar cluster. Our contribution to this scenario is based on the observation that an intermediate SMS can be formed with a subsystem of neutron stars submerged in it. The evolution of neutron star cluster results in its contraction and merging of neutron stars at their collisions. Both these processes have been studied before and rather well known. The merging of neutron stars can produce quasi-spherical fireballs, where protons are accelerated to high energies, and neutrinos are produced.

Described in a more detailed way our scenario looks as follows. The dynamical evolution of a central stellar cluster in the galactic nucleus results in the collision destruction of normal stars and formation of massive gas envelope (Berezinsky $\&$ Dokuchaev 2001) or SMS (this paper). The neutron stars and stellar mass black holes survive through this stage and form selfgravitating subsystem deep inside the SMS. The lifetime of this compact neutron star cluster is very short. The multiple fireballs after neutron star collisions in this cluster dig out a rarefied cavity inside the SMS. The protons are accelerated in the fireballs and in the cavity and produce neutrinos in collisions with an ambient photons and gas. The active phase of high luminosity of the central source has a duration $t \sim 0.1-1$ yr. During the neutrino burst the energy of the same order as a total neutrino energy $\mathcal{E}_{v}$ is released also in the form of high-energy electrons and photons. All high-energy particles, except neutrinos, are completely absorbed in the SMS, which column density can reach $X \sim 10^{9} \mathrm{~g} / \mathrm{cm}^{2}$. Thus the considered object is a very powerful hidden neutrino source. It is demonstrated that that shocks after numerous fireballs are absorbed inside the SMS and cannot destroy it. The system remains gravitationally bound and gravitational collapse of the compact neutron star cluster produces a seed massive black hole inside a host SMS. Long after the neutrino burst, the described hidden neutrino source is to be seen as a bright AGN. As precursors of most powerful AGN, these hidden sources are expected to be at the same redshifts as AGNs. The number of the hidden neutrino sources can be estimated as $\sim 1-10$ in the visible universe.

The hidden neutrino source is detectable by future $1 \mathrm{~km}^{3} \mathrm{de}-$ tector with a number of $\mathrm{TeV}$ muons up to 50-100 per source. The predicted number of low-energy $\left(E_{v}<1 \mathrm{TeV}\right)$ neutrino interactions is $\sim 100$. The highest energy neutrinos with $E>$ $10^{15} \mathrm{eV}$ are probably also detectable.

Acknowledgements. We acknowledge the useful discussions with Bohdan Hnatyk and Yury Eroshenko. We are grateful to anonymous referee for valuable comments. This work was supported in part by the Russian Foundation for Basic Research grants 04-02-16757 and 06-02-16342, and the Russian Ministry of Science grant 1782.2003.2.

\section{References}

Benz, W., \& Hills, J. G. 1992, ApJ, 389, 546 Blandford, R. D., \& McKee, C. F. 1976, Phys. Fluids, 19, 1130 Berezinsky, V. S., \& Smirnov, A. Yu. 1975, Ap. Sp. Sci., 32, 461 Berezinsky, V. 1990, Nucl. Phys. B (Proc. Suppl.), 19, 375

Berezinsky, V. S., Bulanov, S. V., Dogiel, V. A., Ginzburg, V. L., \& Ptuskin, V. S. 1990, Astrophysics of Cosmic Rays, North-Holland, Amsterdam Berezinsky, V. S., \& Dokuchaev, V. I. 2001, Astropart. Phys. 15, 87 Berezinsky, V. S., \& Ginzburg, V. L. 1981, 194, 3

Berezinsky, V. S., \& Prilutsky, O. F. 1987, A\&A, 66, 325 Chandrasekhar, S. 1943, ApJ, 97, 255

Colgate, S. A. 1967, ApJ, 150, 163

Cohn, H. 1980, ApJ, 242, 765

Dokuchaev, V. I. 1991, MNRAS, 251, 564

Dokuchaev, V. I., Eroshenko, Yu. N., \& Ozernoy, L. M. 1998, ApJ, 502, 192

Dokuchaev, V. I. 2002, A\&A, 395, 1023

Gallant Y. A., \& Achterberg, A. 1999, MNRAS, 305, L6

Lai, D., Rasio, F. A., \& Shapiro, S. L. 1993, ApJ, 412, 593 
Landau, L. D., \& Lifshitz, E. M. 1959, Fluid Mechanics, Addison-Wesley Reading, Mass., Chapters X, Sect. 106 and XV

Lemoine, M., \& Revenue, B. 2005 [arXiv:astro-ph/0510522]

New, K. C. B., \& Shapiro, S. L. 2001, ApJ, 548, 439

Ostriker, E. C. 1999, ApJ, 513, 252

Ostriker, J. P., \& McKee, C. F. 1988, Rev. Mod. Phys., 61,

Piran, T. 2000, Phys. Rep., 333, 529

Quinlan, C. D., \& Shapiro, S. L. 1987, ApJ, 321, 199

Quinlan, C. D., \& Shapiro, S. L. 1990, ApJ, 356, 483

Rachen J. P., \& Meszaros, P. 1998, Phys. Rev. D, 58, 123005 [arXiv:astro-ph/9811266]

Rees, M. J. 1984, ARA\&A, 22, 471

Sanders, R. H. 1970, ApJ, 162, 791

Saslaw, W. C. 1987, Gravitational physics of stellar and galactic systems (Cambridge: Cambridge Univ. Press)

Shapiro, S. L., \& Teukolsky, S. A. 1983, Black Holes, White Dwarfs and Neutron Stars (New-York: Willey)
Spitzer, L. 1987, Dynamical Evolution of Globular Clusters (Princeton: Princeton Univ. Press)

Spitzer, L. 1971, Galactic Nuclei, D. Q'Connel, North Holland, Amsterdam, 443 Spitzer, L., \& Saslaw, W. C. 1966, ApJ, 143, 400

Stecker, F. W., Done, C., Salamon, M. H., \& Sommers, P. 1991, Phys. Rev. Lett., 66, 2697

Vietri, M. 1995, ApJ, 453, 883

Waxman, E. 1995, Phys. Rev. Lett., 75, 386

Waxman, E. 2001, Lect. Notes Phys., 576, 122 [arXiv:astro-ph/0103186]

Waxman, E. 2004, ApJ, 606, 988

Waxman, E., \& Bahcall, J. 1997, Phys. Rev. Lett., 78, 2292

Thorne, K. S., \& Zytkow, A. N. 1977, ApJ, 212, 832

Zel'dovich, Ya. B., \& Novikov, I. D. 1971, Relativistic Astrophysics, Vol. 1 (Chicago: Univ. Chicago Press)

Zel'dovich, Ya. B., \& Poduretz, M. A. 1965, Soviet Astr., 9, 742 